Revista Española de Antropología Americana ISSN: 0556-6533

http://dx.doi.org/10.5209/reaa.70367

\title{
¿Textiles mediáticos? Investigar sobre activismo indígena en Panamá, Guatemala y el espacio Web 2.0
}

\author{
Gemma Celigueta Comerma ${ }^{1}$ y Mònica Martínez Mauri²
}

Recibido: 2 de julio de 2020 / Aceptado: 23 de julio de 2020

Resumen. Este artículo analiza las denuncias de apropiaciones culturales derivadas del uso indebido de diseños tradicionales por parte de organizaciones y personas indígenas a través de los medios de comunicación, sobre todo en las redes sociales. A partir de un trabajo de campo multisituado realizado en Panamá, Guatemala y en las redes sociales, estudiamos dos casos: 1) la apropiación por parte de empresas panameñas y multinacionales del arte textil guna protegido por leyes nacionales sui generis de propiedad intelectual y 2) las demandas de protección de los tejidos mayas por parte del Movimiento Nacional de Tejedoras Ruchajixik ri qana'ojbäl de Guatemala. Gracias a nuestro trabajo de campo online y offline, reflexionamos sobre las dificultades y las oportunidades que nos ofrecen la articulación de la etnografía digital y el trabajo de campo clásico para entender la comunicación indígena en contextos marcados por el conflicto en torno a la propiedad de los conocimientos y las expresiones tradicionales.

Palabras clave: apropiación cultural; tejidos mayas; mola guna; etnografía virtual; medios de comunicación; redes sociales.

\section{[en] Stolen Designs? Researching Indigenous Activism in Panama, Guatemala and the Web 2.0}

Abstract. This article focuses on analyzing allegations of cultural appropriation arising from the misuse of traditional designs by indigenous organizations and individuals through the media, especially on social media. Based on a multi-situated fieldwork carried out in Panama, Guatemala and on social networks, it aims to study two cases: 1) the appropriation of Guna textile art, protected by national sui generis intellectual property laws, by Panamanian and multinational companies, and, 2) the demands for protection of Mayan textiles by the National Movement of Weavers Ruchajixik ri qana'ojbäl of Guatemala. Thanks to our online and offline fieldwork, this article reflects on the difficulties and opportunities offered by digital ethnography and classic fieldwork for understanding indigenous communication in contexts marked by conflict over the ownership of traditional knowledge and expressions.

Keywords: cultural appropiation; mayan textiles; guna mola; virtual ethnography; media; social media.

Sumario. 1. Apropiaciones de expresiones culturales tradicionales: ¿una nueva moda? 2. Reflexiones metodológicas entre lo online y lo offline. 3. Apropiaciones indebidas de ECTs en Panamá. 4. Denuncias de apropiaciones culturales en Guatemala. 5. Conclusión. 6. Referencias.

\footnotetext{
Universidad de Barcelona. Profesora lectora Serra-Húnter. gceligueta@ub.edu

$2 \quad$ Universidad de Barcelona. Profesora agregada Serra-Húnter. martinezmauri@ub.edu
} 
Cómo citar: Celigueta Comerma, Gemma y Mónica Martínez Mauri. 2020. “¿Diseños mediáticos? Investigar sobre activismo indígena en Panamá, Guatemala y el espacio Web 2.0". Revista Española de Antropología Americana 50: 241-252.

\section{Apropiaciones de expresiones culturales tradicionales: ¿una nueva moda?}

Cada día son más frecuentes las noticias que se hacen eco de lo que se ha dado en llamar "apropiaciones indebidas", "apropiaciones culturales" o incluso "extractivismo intelectual" por parte de diseñadores/as y grandes marcas globales, de Expresiones Culturales Tradicionales (diseños textiles, símbolos o representaciones) creadas colectivamente por pueblos indígenas o comunidades locales. Casos como el de la diseñadora francesa Isabelle Marant acusada de plagiar los bordados de la blusa tradicional de Santa María Tlahuitoltepec (México) ${ }^{3}$, el de la diseñadora española Stella Rittwagen añadiendo complementos a los bolsos wayuu de Colombia o el de la firma londinense Kokon To Zai utilizando diseños chamánicos inuit en sus prendas (Castillo 2017), son denunciados periódicamente en los medios de comunicación, especialmente en las redes sociales.

Expresiones Culturales Tradicionales (ECTs) es un término acuñado por la Organización Mundial de la Propiedad Intelectual (OMPI) para referirse a formas tangibles e intangibles que expresan, comunican o manifiestan conocimientos y culturas tradicionales ${ }^{4}$. Las ECTs incluyen elementos tan diversos como músicas, actuaciones, narraciones, nombres, símbolos, diseños o formas arquitectónicas. Como antropólogas, hace años que venimos constatando que en los contextos etnográficos en los que trabajamos, las acusaciones de apropiaciones culturales más frecuentes son las que tienen que ver con expresiones materiales, sobre todo textiles. El mundo de la moda parece haber encontrado en los diseños indígenas - muchas veces creados con fines rituales- una fuente de inspiración infinita 5 .

Los conflictos relacionados con las apropiaciones culturales no sólo afectan a las ECTs de los pueblos indígenas, como demuestra el caso de los trajes del carnaval de Viana do Bolo (España), supuestamente plagiados por la firma italiana Dolce \& Gabbana ${ }^{6}$. No obstante, de entre todos los casos de acusaciones de plagio que tienen que ver con ECTs, los que afectan a comunidades indígenas parecen ser los más mediáticos. Su indefensión ante las grandes marcas ilustra de manera tan elocuente la lucha entre David y Goliat, que a veces, hasta los medios más convencionales se han hecho eco de las denuncias que algunos indígenas suelen publicar en sus propios y más modestos medios (redes sociales).

3 Sólo en México, durante el periodo comprendido entre el 2012 y el 2017, la ONG Impacto (http://impacto.org. $\mathrm{mx}$ ) contabilizó ocho casos de plagio por parte de grandes marcas como Zara, Mango, Intropia, Rapsodia, Mara Hoffman o Madewell.

4 https://www.wipo.int/tk/en/resources/glossary.html\#48

5 Frente a la amenaza global del plagio, algunas comunidades indígenas están solicitando peritajes y dictámenes de autenticidad de sus creaciones textiles a organismos como el Instituto Nacional de Antropología e Historia de México (Castillo 2020).

6 https://elpais.com/economia/2018/07/07/actualidad/1530973391_924927.html 
En el marco de una investigación en curso sobre medios de comunicación indígenas ${ }^{7}$ y otra ya conclusa sobre propiedad intelectual y expresiones culturales tradicionales $^{8}$, hemos podido constatar que las denuncias relacionadas con apropiaciones culturales de tejidos y diseños tradicionales ocupan un espacio destacado en las redes sociales de comunicadores y organizaciones indígenas. Muchos indígenas (y algunos no-indígenas) comparten estas publicaciones y reaccionan a través de estos medios ante los casos de plagio de expresiones culturales que consideran suyas, no de dominio público. A través de medios como Facebook, Instagram o Twitter, sus denuncias han podido llegar a una mayor audiencia e incluso ser amplificadas por los medios hegemónicos.

En Guatemala, por ejemplo, Angelina Aspuac, una de las líderes del Movimiento Nacional de Tejedoras Ruchajixik ri qana'ojbäl, cuenta que hay "compañeras" extranjeras que solo conocen a través de las redes sociales, que cuando ven ropa o complementos con diseños mayas en grandes almacenes de países como EEUU, toman fotos y se las mandan para que lo denuncien en sus medios sociales. Gracias a nuestra investigación previa en temas de propiedad intelectual y tejidos indígenas, hemos podido constatar sin embargo que, en Panamá, existen apropiaciones culturales indebidas que a veces no aparecen en los medios y que son intencionalmente invisibilizadas por los propios indígenas. María del Carmen Castillo (2020) también evidencia, para el caso de la blusa de Tlahuitoltepec, cómo las narrativas que difundieron los medios no concordaban con aquello que habían decidido o pactado las poblaciones involucradas a través de sus autoridades. Estas presencias y ausencias mediáticas nos han llevado a pensar en cómo el creciente uso de los medios digitales en los estudios de ciencias sociales amplifica, por un lado, nuestro conocimiento sobre las relaciones y auto-representaciones de nuestros sujetos de estudio, pero por el otro, limita la comprensión holística en los estudios etnográficos. Por esta razón dedicaremos la siguiente sección a reflexionar sobre ciertos aspectos metodológicos que han influido de manera significativa en el desarrollo de la presente investigación.

\section{Reflexiones metodológicas entre lo online y lo offline}

Aproximarnos al estudio de las apropiaciones de expresiones culturales tradicionales y su visibilidad -o invisibilidad- en los medios, nos ha obligado a diseñar una estrategia de investigación que contemplara tanto el trabajo de campo online (en línea) como offline (fuera de línea). Para pensar la relación entre estos dos espacios, estudios previos sobre la mediación tecnológica de la comunicación nos han ayudado a comprender que su articulación es más compleja de lo que puede parecer en un primer momento.

En los años 1990, cuando el uso de Internet todavía era muy incipiente, los entornos sociales online fueron conceptualizados y analizados como esferas separadas de los espacios offline (Paccagnella 1997). Durante la última década, sin embargo, ante

Proyecto: “Comunicación indígena y patrimonio cultural en América Latina: conservación, revitalización, creatividad”. Ministerio de Ciencia, Innovación y Universidades (PGC2018-095841-B-I00). 2019-2021. Investigadora principal: Gemma Orobitg Canal.

8 Proyecto: "Etnicidad material: expresiones culturales tangibles y propiedad intelectual". Ministerio de Economía y Competitividad [CSO2015-62723-ERC]. 2015-2016. Investigadora Principal: Mònica Martínez Mauri. 
la expansión global del uso de Internet a través de smartphones y otros dispositivos móviles, varios etnógrafos han abogado por la eliminación de las falsas barreras entre los espacios en línea y fuera de línea en la investigación etnográfica (Gatson y Zweerink 2004). Algunos, como Jurgenson (2012) han cuestionado el "dualismo digital" constatando que actualmente la red digital se combina con los encuentros físicos formando una "realidad aumentada". Aunque los medios no son suficientes para determinar nuestra situación social, en la actualidad forman parte de los condicionantes tecnológicos y ambientales que marcan el devenir humano. Ante este nuevo contexto, el espacio online es incorporado en las investigaciones etnográficas como una dimensión más de la realidad que intentamos aprehender. Ejemplo de ello es el ya clásico estudio de Miller y Slater (2004) sobre la interacción entre el mundo online y offline en cibercafés de Trinidad y Tobago.

Sin embargo, esta mezcla de observaciones online y offline en el trabajo de campo, si bien es cada día más recurrente en los estudios etnográficos (Murthy 2008; García et al. 2009), no ha supuesto un avance significativo en la epistemología del campo digital (Airoldi 2018). De hecho, la mayoría de enfoques etnográficos sobre Internet se han limitado a observar sitios web o comunidades de usuarios (ver Kozinets 2010; Hine 2015). De entre todos los modelos desarrollados para estudiar etnográficamente Internet y las redes sociales -virtual ethnography (Hine 2000), internet ethnography (Miller y Slater 2001), cyber-ethnography (Escobar 1994), expanded ethnography (Beneito-Montagut 2011), ethnography of the virtual worlds (Boellstorff et al. 2012), ethnography for the internet (Hine 2015); netnography (Kozinets 2010), cyberethnography (Robinson y Schulz 2009), ethnography of virtual spaces (Burrell 2009) e internet-related ethnography (Postill y Pink 2012)- ninguno parece ser del todo válido para entender con claridad el papel de Internet en la conformación o modificación de los sistemas sociales. La mayoría de estos enfoques, como el de etnografía para el Internet (Caliandro 2017), parecen útiles para mapear los entornos online de los internautas pero sin mantener una interacción directa con ellos. Aunque estas investigaciones son eficaces para obtener nuevos datos que permitan entender parcialmente las formaciones sociales, los sistemas de significado y las estrategias de auto-presentación, no parecen suficientes para analizar la influencia de los medios en las formas de sociabilidad de áreas periféricas, como las indígenas, alejadas de los centros urbanos, con acceso desigual a los recursos tecnológicos, y que se comunican a través de lenguas minoritarias.

Tal y como ya apuntó Coleman (2010), inspirándose en Chakrabarty (Chakrabarty 2000 en Coleman 2010: 489) "provincializar" los medios digitales sigue siendo una tarea pendiente para la antropología. Gracias a nuestra capacidad de interacción presencial, los antropólogos podemos entender cómo los medios -sin negar su alcance global-se han vuelto centrales en la articulación de creencias, prácticas rituales y modos de estar en el mundo. Nuestra tarea es entender cómo, dónde y por qué la experiencia digital es importante poniéndola en relación con los contextos offline. Al igual que articulamos el pasado con el presente, tenemos que ser capaces de mostrar significados y usos paradójicos de estos nuevos medios, como, por ejemplo, que las redes sociales pueden tanto promover las movilizaciones populares como los discursos de odio, la vigilancia gubernamental y las violaciones de los derechos humanos (Coleman 2010: 493).

Aunque la antropología ha entrado en los espacios digitales de muchas sociedades, una década después, las críticas de Ginsburg (2008), retomadas por la misma 
Coleman (2010), siguen siendo válidas. Todavía seguimos dominados por la idea de que los Otros viven en una dimensión temporal distinta (allochronic chronopolitics) y se utiliza la era digital para construir nuevos muros y alteridades parecidos a los de las teorías de la modernización. Se habla de las sociedades indígenas como si se hubieran quedado al margen de este mundo dominado por los medios digitales (López García 2012). Aunque muchos indígenas están utilizando aplicaciones como Facebook, WhatsApp o Instagram, los académicos no se han interesado demasiado por el uso que les están dando las sociedades llamadas tradicionales.

En nuestro caso, nos ha sido útil partir de la idea que estamos ante una investigación multisituada (Marcus 1995). Tal y como han hecho otros investigadores interesados en incluir los espacios virtuales en sus pesquisas (Hallett y Barber 2014; Caliandro 2017), hemos concebido las redes sociales (sobre todo los perfiles de Facebook y grupos de WhatsApp) de nuestros interlocutores como un nuevo espacio en el que seguirles, incluso cuando no estamos con ellos presencialmente. De hecho, uno de los grandes potenciales de los espacios virtuales reside justamente en la posibilidad de seguir conectados con nuestros interlocutores desde la distancia a la vez que interactuamos, literalmente, con sus redes sociales, complementando el llamado muestreo de bola de nieve. Estas redes -también conocidas como Web 2.0 porque a diferencia de la 1.0 permiten la interacción de los usuarios con los contenidos y otros usuarios online (O'Reilly 2005) - nos han permitido observar cómo las personas con las que habíamos establecido rapport previamente- se comunican, transmiten información y colaboran en línea con otras personas, algunas de las cuales también comparten algún tipo de relación offline entre ellas y buena parte de sus contactos. Es por ello que no debemos entender el acompañamiento de nuestros interlocutores locales en el mundo de las redes sociales en detrimento de las interacciones presenciales previas y a la inversa. Internet es una dimensión más de la vida cotidiana, no una realidad alterna. Una tecnología que utilizan constantemente para potenciar sus identidades, así como sus vínculos y actividades sociales (Hine 2000; Woolgar 2002). Tanto en las comunidades indígenas de Guatemala como en las de Panamá con las que venimos trabajando desde hace años, separar el espacio online del offline es ilusorio. Y a medida que las actividades en estos dos espacios se van fusionando, ambos también interactúan y se transforman entre sí (García et al. 2009: 53).

Pero ¿cómo hemos aplicado una perspectiva etnográfica al estudio de las apropiaciones de expresiones culturales tradicionales denunciadas en los medios de comunicación? En nuestro caso, la perspectiva etnográfica ha implicado primero, un trabajo de campo previo de largo recorrido basado en la observación participante y las entrevistas en profundidad que nos ha proporcionado una base de conocimientos y relaciones sociales en la región. Segundo, trabajos de campos puntuales sobre la temática en cuestión que nos han permitido establecer nuevas relaciones y profundizar en otras más antiguas, así como observar y participar en actos relacionados con las apropiaciones de ECTs. Y tercero, un trabajo de seguimiento de la información disponible en Internet y de seguimiento a nuestros interlocutores locales en los espacios virtuales para observar lo que comparten, pero también lo que no muestran.

En el tema que nos ocupa, el de las denuncias indígenas de apropiaciones culturales, ha sido fundamental complementar el panorama que se dibujaba a través de los medios, con observación y realización de entrevistas presenciales que nos han permitido documentar lo que pasaba entre bambalinas: en el caso guna, las negocia- 
ciones discretas, acordes con la agenda política de los diferentes actores, que en la mayoría de los casos culminaban en acuerdos de confidencialidad. En el caso maya, la preocupación por sincronizar las denuncias de apropiación cultural y racismo presentadas por el Movimiento Nacional de Tejedoras Ruchajixik ri qana'ojbäl con los intereses de su agenda política.

\section{Apropiaciones indebidas de ECTs en Panamá}

Panamá fue el primer país de América Latina ${ }^{9}$ y del mundo en desarrollar una legislación sui generis para proteger las expresiones culturales tradicionales de los pueblos indígenas que se encontraban dentro de sus fronteras. En el año 2000 se aprobó la Ley 20 sobre "el régimen especial de propiedad intelectual sobre los derechos colectivos de los pueblos indígenas, para la protección y defensa de su identidad cultural y de sus conocimientos tradicionales". Gracias a esta norma, un sistema especial de registro - en el Departamento de Derechos Colectivos y Expresiones Folclóricas de la Dirección General del Registro de Propiedad Industrial (DIGERPI) del Ministerio de Comercio e Industrias- da protección a las invenciones, modelos gráficos, petroglifos, símbolos, figuras, dibujos, diseños u otros detalles indígenas. A diferencia de lo que ha sucedido en otros países de la región, en Panamá los indígenas pueden impedir que los elementos que han registrado como suyos sean utilizados con fines comerciales por empresas nacionales. En 2001, los gunas, con el registro de la mola - composiciones textiles elaboradas a partir de la técnica del aplicado ${ }^{10}$ fueron los primeros indígenas panameños en hacer uso de esta instancia. Desde su aprobación en 2002, el Congreso General de Gunayala fue la entidad responsable de entregar los permisos de reproducción total o parcial de la mola a partir de licencias de uso del derecho colectivo indígena registrado bajo el nombre Mola (morra) Kuna Panamá (Valiente 2006: 128-136). A partir del año 2016 esta competencia es compartida por los cuatro entes políticos del pueblo guna en Panamá: Congreso General de Gunayala, Congreso General de Wargandi, Congreso General de Madungandi y Consejo del Territorio Ancestral de Tagargunyala. Con la entrega de estas licencias, empresas no indígenas pueden hacer uso de los diseños reproducidos en las molas a cambio del pago de regalías a las autoridades indígenas. Además de la Ley 20, los gunas de Panamá cuentan con otro aliado legal para proteger su arte. El código penal de 2007 (Ley 14 de 18 de mayo) incorpora el delito contra derechos colectivos de los pueblos indígenas. Esto significa que quien fabrique o comercialice molas sin el consentimiento de las autoridades indígenas se expone a penas de cuatro a seis años de prisión.

A pesar de este marco legal tan favorable, en Panamá se han dado numerosos casos de apropiaciones indebidas, sobre todo en relación a la mola registrada por los gunas. Sus coloridos diseños han sido reproducidos en estuches, afiches anunciando programas de televisión o botellas de ron y cerveza. Curiosamente estas transgresio-

\footnotetext{
El año 2016, Ecuador aprobó legislación sui generis para proteger las ECTs, concretamente el Código orgánico de la economía social de los conocimientos, creatividad e innovación. En 2019, México también tomó medidas parecidas aprobando una Ley General de Salvaguardia de los Elementos de la Cultura e Identidad de los Pueblos y Comunidades Indígenas, Afromexicanas y Equiparables.

10 Técnica parecida al patchwork que consiste en coser diversas capas de tejido superpuestas.
} 
nes de la Ley 20 no han suscitado mucho revuelo en las redes sociales. En algunas ocasiones han sido señaladas por algunos activistas indígenas, pero de forma muy discreta. De hecho, estos casos no han sido ni publicados en los periódicos nacionales ni comentados en los noticieros televisivos o radiales. Lo que ha pasado en estas ocasiones es que de forma muy rápida y discreta los asesores de las autoridades indígenas han iniciado negociaciones con las empresas panameñas que habían distribuido productos que incluían diseños de mola sin su permiso. Rápidamente les han informado que si no querían retirar del mercado todos los artículos que ya habían distribuido -con el elevado coste que esto comportaría-, deberían contar con su autorización. Llegados a este punto, después de valorar los costes, algunas empresas han preferido retirar los diseños de mola o negociar un pago para mantenerlos.

Con todo es evidente que la Ley 20 supone un obstáculo para las apropiaciones indebidas de la mola en Panamá. De hecho, muchas de ellas acaban en negociaciones discretas que contemplan cláusulas de confidencialidad que impiden que estos casos lleguen a los medios. Esta situación ilustra muy bien uno de los problemas que suscita centrar las etnografías exclusivamente en los espacios online. Sin hacer trabajo de campo convencional no hubiéramos podido documentar la existencia de estos casos de plagio y las negociaciones entre bambalinas en torno a los diseños indígenas.

La pregunta que queda por responder es: ¿qué denuncias aparecen en los medios gunas? En las redes sociales como Facebook se suelen comentar las apropiaciones indebidas de empresas que operan fuera del marco normativo panameño, es decir, las que se producen fuera de las fronteras nacionales. Un ejemplo reciente fue el uso de una mola en un modelo de zapatilla deportiva de la conocida marca estadounidense Nike. El caso dio la vuelta al mundo en mayo de 2019, cuando la empresa comunicó, a través de las redes sociales, que en junio lanzaría al mercado un nuevo modelo de vistosos colores, dedicado a Puerto Rico. El anuncio venía acompañado de una imagen de la zapatilla en la que se podía apreciar una colorida mola sin que la marca se hubiera referido a ella ni a sus productores. Rápidamente aparecieron voces críticas, entre ellas de activistas indígenas norteamericanos, con la multinacional, a la que acusaron de plagio y de usar de forma descontextualizada un modelo indígena sin dar crédito a los verdaderos creadores del diseño.

En Panamá la noticia circuló rápidamente por Facebook, Twitter, Instagram y grupos de WhatsApp. Todos, indígenas y no indígenas, se indignaron al ver que la marca no reconocía la procedencia del diseño. Las cuatro naciones gunas hicieron circular un comunicado en el que dejaban claro que la multinacional había usado la mola sin consentimiento, "solo con el afán de generar millones de dólares a espaldas de nuestras madres, hermanas gunas que por siglos han preservado este conocimiento tradicional colectivo"11. Con todo, las autoridades gunas convocaron una rueda de prensa el 21 de mayo, con el apoyo del Ministerio de Comercio e Industria de Panamá $^{12}$. Al cabo de pocas horas de comparecer en público, los medios internacionales se hicieron eco de la noticia y Nike anunció que cancelaba el lanzamiento del modelo Puerto Rico ${ }^{13}$.

https://www.facebook.com/photo?fbid=680175092452378\&set=pcb.680175112452376.

12 Véase noticia sobre la rueda de prensa en: http://mediosindigenas.ub.edu/2019/05/20/panama-convocatoria-de-prensa-del-congreso-general-guna-en-relacion-a-la-comercializacion-de-zapatillas-con-motivos-del-pueblo-guna-por-la-empresa-nike/.

13 Véase noticia dedicada a la polémica en: http://mediosindigenas.ub.edu/2019/05/14/panama-conversatorio-so- 
A la hora de analizar el papel que jugaron los medios en la retirada del producto por parte de Nike es inevitable preguntarse qué hubiera pasado sin el ruido provocado a través de las redes. La primera cuestión que llama la atención es que no fueron las autoridades indígenas las que empezaron a acusar a la multinacional, sino que fueron ciudadanos norteamericanos, algunos de ellos activistas indígenas, los que lanzaron las primeras acusaciones. Sin este ruido mediático y los llamados a actuar, las autoridades gunas hubieran preferido sentarse a negociar, ya que sabían que el marco legal también les era favorable. Y es que gracias a la firma del Tratado de Promoción Comercial (TPC) entre Panamá y Estados Unidos, la Ley 20 que da protección a la mola, es de obligado cumplimiento en ambos países. Esto significa que la multinacional no hubiera tenido nada que hacer frente a una posible demanda del pueblo guna.

\section{Denuncias de apropiaciones culturales en Guatemala}

En Guatemala, a diferencia de Panamá, no existe ninguna ley que proteja las expresiones culturales tradicionales mayas. Con el fin de subsanar este vacío legal, las integrantes de la Asociación Femenina para el Desarrollo de Sacatepéquez (AFEDES) han presentado una acción de inconstitucionalidad por omisión de normas que protejan los textiles y la indumentaria maya (2016) y la iniciativa de ley 5247 (2017), que busca reformar las leyes de derechos de autor y de la propiedad industrial. También han estado trabajando en la elaboración de una ley específica para la protección de las expresiones culturales tradicionales, siguiendo el ejemplo de la Ley 20 de Panamá. En el caso de ser aprobada, se podrían establecer mecanismos para regular el uso de los diseños mayas por parte de terceros y la posibilidad de sancionar a quien no cumpliera con los mismos. AFEDES también ha liderado la constitución del Movimiento Nacional de Tejedoras Ruchajixik ri qana'ojbäl así como de varias escuelas de tejidos y comités locales de tejedoras en diferentes comunidades del país. La principal finalidad de dichos comités es la protección de las expresiones culturales tradicionales de su propia comunidad, especialmente los diseños de los tejidos. Si la ley fuera aprobada, estos comités serían los responsables de otorgar los permisos de reproducción y administrar los derechos de autor ${ }^{14}$.

Pero como reconocen las mismas tejedoras, es muy difícil que en Guatemala se apruebe ninguna ley específica sobre pueblos indígenas como sí ha ocurrido en Panamá. Ante esta situación, "que se hable de ello", es decir, crear debate y tener la oportunidad de exponer sus argumentos sobre la necesidad de protección de los tejidos mayas, ha sido uno de los principales objetivos de su movilización.

Por ello, sus acciones han ido acompañadas de la correspondiente difusión mediática, ya sea a través de entrevistas que conceden a medios digitales y/o convencionales o de sus propias redes sociales, especialmente Facebook. Una de las estrategias de comunicación de las tejedoras ha sido la denuncia, en las redes sociales, de empresas nacionales e internacionales que utilizan diseños mayas en sus creaciones. En algunas ocasiones, las tejedoras han elevado estas denuncias hasta instituciones

bre-usos-y-retos-de-los-medios-de-comunicacion-indigenas/.

14 Véase la noticia en: http://mediosindigenas.ub.edu/2017/11/04/tras-anos-de-apropiacion-cultural-tejedoras-mayas-quieren-proteger-su-patrimonio/. 
como la Procuraduría de los Derechos Humanos de Guatemala (PDHG) o la Comisión Presidencial contra la Discriminación y el Racismo (CODISRA), logrando ampliar así su audiencia: no tanto para obtener una sanción legal -puesto que no existe legislación al respecto- sino para conseguir una condena moral que difunda su causa. Por ejemplo, Angelina Aspuac, una de sus líderes, cuenta como caso de éxito la disculpa pública - es decir, ante los medios- del diseñador guatemalteco César Alejandro Portillo por usar el traje de San Juan Sacatepéquez en una de sus creaciones. Ante la denuncia en su contra que algunas mujeres del Movimiento interpusieron en la PDHG, el diseñador no sólo se disculpó, sino que dijo que "quería entender y respetar" y que por eso invitaba a leer las columnas de opinión en el periódico $L a$ Hora de la antropóloga, periodista y también activista del Movimiento de Tejedoras, Sandra Xinico.

En otras ocasiones, ha sido gracias a la presión de las redes sociales que una institución estatal como la CODISRA ha reaccionado y denunciado a una de esas empresas. Ése fue el caso de la denuncia a la tienda nacional de venta de ropa y complementos fabricados con tejidos mayas María Chula. Al igual que habían hecho en otras ocasiones y con empresas distintas; líderes y simpatizantes de las tejedoras solicitaron a CODISRA que investigara la tienda cuyo nombre, asociado a las mujeres indígenas y sus creaciones textiles, interpretaron como racista ${ }^{15}$. A finales de mayo del 2017, CODISRA presentó la denuncia por posible caso de discriminación al Ministerio Público "derivado del análisis de contexto del nombre de la tienda virtual y las prendas que se comercializan ya que los términos María Chula, han sido utilizados como parte del lenguaje discriminatorio hacia las mujeres mayas" (comunicado de CODISRA, 17/07/2017). No se mencionó la apropiación cultural, que no constituye delito en Guatemala, como parte del problema.

Como se había hecho también en otras ocasiones, CODISRA acordó desestimar la denuncia a cambio de que la propietaria de la tienda pidiera disculpas públicamente en un acto de conciliación que se realizó el 17 de julio, en su misma sede y ante los medios de comunicación. Esta vez, sin embargo, la disculpa pública de la joven dueña de María Chula cayó como jarro de agua fría sobre muchos guatemaltecos, sobre todo no-indígenas, que sintieron dicha disculpa como una humillación propia. Las críticas e insultos en contra de CODISRA y aquellas que habían promovido la denuncia, como las líderes del Movimiento Nacional de Tejedoras, se hicieron rápidamente virales ${ }^{16}$. Y aunque ellas intentaron aprovechar la ocasión para volver a plantear sus argumentos sobre la apropiación cultural, el despojo a las sociedades indígenas o la naturalización del racismo en la sociedad guatemalteca; la controversia

15 En Guatemala (y también en México), el nombre de María se utiliza para llamar de forma peyorativa a las mujeres indígenas. El nombre se asocia a una mujer vestida con el traje indígena y que además trabaja como criada o sirvienta.

16 Además del hashtag \#yoapoyoaMariaChula que apareció en las redes sociales de muchos guatemaltecos, abundaron las acusaciones de "racistas al revés", resentidos, abusivos e hipócritas, incluso en varios artículos de opinión de los principales diarios del país. Véanse, por ejemplo, los 206 comentarios al comunicado de CODISRA sobre el caso de María Chula, colgado el 17 de julio del 2017 en su propio muro de Facebook (https:// www.facebook.com/codisra.comisionpresidencial/photos/a.672070629521722/1677908505604591/?type=3\&theater). Véanse también los artículos de opinión: https://elperiodico.com.gt/opinion/2017/07/21/la-importancia-de-maria-chula/; https://elsiglo.com.gt/2017/07/21/black-pitaya-la-mariachula/; https://www.prensalibre. com/opinion/maria-nuevo-insulto/; https://elsiglo.com.gt/2017/07/21/racismo-o-apropiacion-cultural/; https:// lahora.gt/maria-chula-la-cultura-del-odio/. 
derivó en un rifirrafe cuyo ruido mediático acalló las posibilidades del debate. Todos parecen estar de acuerdo en ello. La controversia mediática de María Chula no solo mostró los límites de la comunicación sino también su fracaso, plagado de insultos, burlas y malentendidos.

La polémica sobre María Chula dejó un sabor agridulce en el Movimiento Nacional de Tejedoras. "Nos sobrepasó", reconoce Angelina. Y aunque las tejedoras reafirmaron la importancia de las denuncias en los medios y en los tribunales en la lucha global por los derechos indígenas, también se mostraron preocupadas por los efectos negativos que la disputa mediática podía causar en su lucha política así como por el diálogo de sordos que había generado la controversia. “¿Por qué hay gente que se ofende cuando denunciamos cosas que nos ofenden a las mujeres mayas?" Con este mensaje en su página personal de Facebook, Angelina Aspuac expresaba su desconcierto ante las reacciones provocadas por el caso de María Chula. Cuando un mes después las avisaron de que el Congreso de los diputados iba a dar una primera lectura a la iniciativa de ley de las tejedoras, éstas decidieron no convocar a los medios. "Mejor no hagamos bulla... solo un poco de publicidad en las redes" -se dijeron, conscientes de que, en Guatemala, la controversia mediática de María Chula podía perjudicar a su causa-.

\section{Conclusión}

Los casos expuestos sobre denuncias de apropiaciones culturales en Panamá y Guatemala muestran cómo el contexto legal y político condiciona su visibilidad - o invisibilidad- en los medios. Ambos casos también demuestran como la visibilidad en los medios no siempre es deseada y puede volverse incluso problemática. Finalmente, ambos ejemplos ilustran modos diferentes de denunciar la apropiación cultural así como la necesidad compartida de articular, para su comprensión por parte de las investigadoras, sus universos online y offline.

Tal y como sostienen Campanella y Barros (2016) cuando se habla de aplicar una "perspectiva etnográfica al estudio de los medios", sobre todo de Internet, la palabra etnografía se vuelve muy polisémica. Nuestra experiencia en el análisis de denuncias de apropiaciones culturales derivadas del uso de diseños tradicionales indígenas a través de los medios de comunicación nos lleva a concluir que la investigación etnográfica de largo recorrido, fundamentada en la observación participante y las entrevistas en profundidad, sigue siendo la más adecuada para descifrar algunas facetas veladas del uso y propagación de los medios digitales (Coleman 2010).

Al igual que ha sucedido en otros contextos culturales, la distinción entre mundos indígenas online y offline se está diluyendo. Este nuevo contexto nos obliga a replantear nuestro quehacer etnográfico, pero no a abandonar el trabajo de campo presencial. Si combinamos la investigación online-offline, podemos apreciar como los medios de comunicación en general y los sociales en particular son utilizados como una herramienta de mediación y denuncia por parte de los pueblos indígenas, pero también como a veces prefieren obviar este tipo de mediación en función del contexto legal -más favorable en Panamá que en Guatemala-y de sus propias agendas políticas. Incluso, en futuras investigaciones centradas en entender los usos de redes sociales y el impacto que han tenido éstas en las dinámicas indígenas, sería oportuno documentar cómo se han visto transformados sus propios sistemas de gobierno. 
A partir de los casos que hemos presentado, se hace evidente que tanto para entender las omisiones de apropiaciones indebidas de la mola en los medios gunas como para comprender el alcance de las denuncias relacionadas con los tejidos mayas, es necesario relacionarlas con los agentes que las promueven y el contexto sociopolítico en el que surgen. Y esto solo podemos hacerlo creando rapport con los actores locales y prestando atención etnográfica a todos los aspectos de su realidad, tanto en las redes como fuera de ellas.

\section{Referencias}

Airoldi, Massimo. 2018. «Ethnography and the Digital Fields of Social Media». International Journal of Social Research Methodology 21 (6): 661-673. https://doi.org/10.1080/13645579.2018.1465622.

Beneito-Montagut, Roser. 2011. «Ethnography Goes Online: Towards a User-Centered Methodology to Research Interpersonal Communication on the Internet». Qualitative Research 11: 716-375. https://doi.org/10.1177/1468794111413368.

Boellstorff, Tom, Bonnie Nardi, Celia Pearce y T. L. Taylor. 2012. Ethnography and Virtual Worlds: A Handbook of Method. Princeton: Princeton University Press.

Burrell, Jenna. 2009. «The Field Site as a Network: a Strategy for Locating Ethnographic Research». Field Methods 21 (2): 181-199. https://doi.org/10.1177/1525822X08329699.

Caliandro, Alessandro. 2017. «Digital Methods for Ethnography: Analytical Concepts for Ethnographers Exploring Social Media Environments». Journal of Contemporary Ethnography 47 (5): 551-578. https://doi.org/10.1177/0891241617702960.

Campanella, Bruno y Carla Barros. 2016. Etnografia e consumo midiático. Novas tendências e desafios metodológicos. Río de Janeiro: E-papers.

Castillo, M. Carmen. 2020. «Hilar memorias para tejer historia: Hacia una antropología textil en Oaxaca». Revista Euroamericana de Antropología 9: 125-139. https://doi.org/10.14201/rea20209125139

Castillo, María del Carmen. 2017. «La blusa de Tlahuitoltepec Xaam nïxuy es identidad», en Acervo Mexicano: Legado de culturas, Erika Galicia Isasmendi, Fernando Quiles García y Zara Ruiz Romero, eds., pp. 170-191. México: Universidad Autónoma de Puebla.

Coleman, E. Gabriella. 2010. «Ethnographic Approaches to Digital Media». Annual Review of Anthropology 39 (1): 487-505. https://doi.org/10.1146/annurev.anthro.012809.104945.

Escobar, Arturo. 1994. «Welcome to Cyberia». Current Anthropology 35 (3): 211-231. https://doi.org/10.1086/204266.

García, Ángela Cora, Alecea I. Standlee, Jennifer Bechkoff y Yan Cui. 2009. «Ethnographic Approaches to the Internet and Computer-Mediated Communication». Journal of Contemporary Ethnography 38: 52-84. https://doi.org/10.1177/0891241607310839.

Gatson, Sarah N. y Amanda Zweerink. 2004. «Ethnography Online: 'Natives' Practicing and Inscribing Community». Qualitative Research 4: 179-200. https://doi.org/10.1177/1468794104044431.

Ginsburg, Faye. 2008. «Rethinking the Digital Age», en The Media and Social Theory, David Hesmondhalgh y Jason Toynbee, eds., pp. 127-144. Londres y Nueva York: Routledge.

Hallett, Ronald E. y Kristen Barber. 2014. «Ethnographic Research in a Cyber Era». Journal of ContemporaryEthnography 43(3):306-330. https://doi.org/10.1177/0891241613497749.

Hine, Christine. 2000. Virtual Ethnography. Londres: Sage Publications. 
-. 2015. Ethnography for the Internet: Embedded, Embodied and Everyday. Londres: Bloomsbury.

Jurgenson, Nathan. 2012. «When Atoms Meet Bits: Social Media, the Mobile Web and Augmented Revolution». Future Internet 4 (1): 83-91. https://doi.org/10.3390/fi4010083.

Kozinets, Robert. 2010. Netnography: Doing Ethnographic Research Online. Londres: Sage Publications.

López García, Julián. 2012. «Teléfonos celulares en la era de los mayas: representaciones y usos entre los ch'ort'i de Guatemala», en Modernidades indigenas, Pedro Pitarch y Gemma Orobitg, eds., pp. 89-114. Madrid: Iberoamericana/Vervuert.

Marcus, George E. 1995. «Ethnography in/of the World System: The Emergence of Multi-sited Ethnography». Annual Review of Anthropology 24: 95-117. https://doi.org/10.1146/annurev.an.24.100195.000523.

Miller, Daniel y Don Slater. 2001. The Internet: An Ethnographic Approach. Londres: Berg Publishers.

—. 2004. «Etnografia on e off-line: cibercafés em Trinidad». Horizontes antropológicos 21: 41-65. https://doi.org/10.1590/S0104-71832004000100003.

Murthy,Dhiraj.2008. «DigitalEthnography:AnExamination of theUseofNew Technologies for Social Research». Sociology 42 (5): 837-855.https://doi.org/10.1177/0038038508094565.

O'Reilly, Tim. 2005. «What Is Web 2.0». O'Reilly Media, 30 Septiembre. http://www.oreilly.com/pub/a/web2/archive/what-is-web-20.html.

Paccagnella, Luciano. 1997. «Getting the Seats of Your Pants Dirty: Strategies for Ethnographic Research on Virtual Communities». Journal of Computer Mediated Communication 3 (1): 1-17. https://doi.org/10.1111/j.1083-6101.1997.tb00065.x.

Postill, John y Sarah Pink. 2012. «Social Media Ethnography: the Digital Researcher in a Messy Web». Media International Australia 145: 123-134. https://doi.org/10.1177/1329878X1214500114.

Robinson, Laura y Jeremy Schulz. 2009. «New Avenues for Sociological Inquiry: Evolving Forms of Ethnographic Practice». Sociology 43: 685-698. https://doi.org/10.1177/0038038509105415.

Valiente, Aresio. 2006. Régimen jurídico de protección a la mola como manifestación cultural. Tesis de Licenciatura. Universidad de Panamá.

Woolgar, Steve. 2002. Virtual Society?: Technology, Cyberbole, Reality. Oxford: Oxford University Press. 